\title{
Crystallization of $\mathrm{CO}_{2}$ ice and the absence of amorphous $\mathrm{CO}_{2}$ ice in space
}

\author{
Rafael M. Escribano ${ }^{a, 1}$, Guillermo M. Muñoz Caro ${ }^{b, 1}$, Gustavo A. Cruz-Diaz ${ }^{b}$, Yamilet Rodríguez-Lazcano ${ }^{a}$, \\ and Belén Maté
}

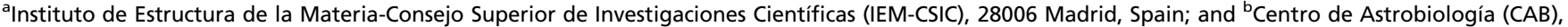
Instituto Nacional de Técnica Aeroespacial-Consejo Superior de Investigaciones Científicas (INTA-CSIC), Torrejón de Ardoz, 28850 Madrid, Spain

Edited by Neta A. Bahcall, Princeton University, Princeton, NJ, and approved June 21, 2013 (received for review December 20, 2012)

Carbon dioxide $\left(\mathrm{CO}_{2}\right)$ is one of the most relevant and abundant species in astrophysical and atmospheric media. In particular, $\mathrm{CO}_{2}$ ice is present in several solar system bodies, as well as in interstellar and circumstellar ice mantles. The amount of $\mathrm{CO}_{2}$ in ice mantles and the presence of pure $\mathrm{CO}_{2}$ ice are significant indicators of the temperature history of dust in protostars. It is therefore important to know if $\mathrm{CO}_{2}$ is mixed with other molecules in the ice matrix or segregated and whether it is present in an amorphous or crystalline form. We apply a multidisciplinary approach involving IR spectroscopy in the laboratory, theoretical modeling of solid structures, and comparison with astronomical observations. We generate an unprecedented highly amorphous $\mathrm{CO}_{2}$ ice and study its crystallization both by thermal annealing and by slow accumulation of monolayers from the gas phase under an ultrahigh vacuum. Structural changes are followed by IR spectroscopy. We also devise theoretical models to reproduce different $\mathrm{CO}_{2}$ ice structures. We detect a preferential in-plane orientation of some vibrational modes of crystalline $\mathrm{CO}_{2}$ We identify the IR features of amorphous $\mathrm{CO}_{2}$ ice, and, in particular, we provide a theoretical explanation for a band at $2,328 \mathrm{~cm}^{-1}$ that dominates the spectrum of the amorphous phase and disappears when the crystallization is complete. Our results allow us to rule out the presence of pure and amorphous $\mathrm{CO}_{2}$ ice in space based on the observations available so far, supporting our current view of the evolution of $\mathrm{CO}_{2}$ ice.

astrochemistry | solid state morphology

C arbon dioxide $\left(\mathrm{CO}_{2}\right)$ has come to play a fundamental role in several aspects of the Earth's geophysics $(1,2)$, but it is also a key element in astrophysical research $(3,4)$. In the interior of dense interstellar clouds, as well as in the envelopes around young stars, dust grains are covered by ice mantles formed by frozen volatile molecules, with water being the most abundant molecular species, followed by carbon monoxide (CO), $\mathrm{CO}_{2}$, methanol, methane, and others $(5,6)$. The structure of $\mathrm{CO}_{2}$ in the icy phase of the interstellar grains is still an open question. Is $\mathrm{CO}_{2}$ mixed up with other frozen components, or is it segregated in multilayer structures (7)? Has it attained a crystalline arrangement, or does it have an amorphous structure (8)? Because solid $\mathrm{CO}_{2}$ is an indicator of the temperature history in the envelopes of young stars $(9,10)$, it is important to address these questions. Most of the available information on these systems comes from spectroscopic observations. Thus, many laboratory experiments have been performed on low-temperature $\mathrm{CO}_{2}$, both as a single species and mixed with other components, using IR spectroscopy as the main detection tool (11-15). In the context of solid-state physics, the existence of transverse optical (TO) and longitudinal optical (LO) modes in amorphous materials was questioned because the origin of this effect was linked to long-range order in crystals, but it was proved that longitudinal modes can also propagate in amorphous media (16). We provide experimental and theoretical evidence of LO/TO splitting in the spectra of amorphous $\mathrm{CO}_{2}$ ice.

The formation of the $\mathrm{CO}_{2}$ observed in ice mantles is expected to occur through reactions on and in the ice matrix. These $\mathrm{CO}_{2}$ molecules will desorb eventually, and the grains may accrete, forming new ice mantles, provided that the dust temperatures are low enough. This contribution deals with the process undergone by amorphous $\mathrm{CO}_{2}$ when transforming to its crystalline structure. We will show that the energy delivered on the amorphous solid by heating the substrate that holds it is equivalent to that originating from the accumulation of successive layers simulating $\mathrm{CO}_{2}$ accretion on dust, as far as the crystallization process is concerned, although complete crystallization is only reached after thermal annealing. Our tools are different varieties of IR spectroscopy supported by computer models of both amorphous and crystalline $\mathrm{CO}_{2}$. Our materials are slowly grown ices of $\mathrm{CO}_{2}$ at cryogenic temperatures as low as $8 \mathrm{~K}$ and with an ultrahigh vacuum. At the same time, we propose answers to two specific questions often raised in the astrophysics and ice physics communities: How can we distinguish between pure and amorphous $\mathrm{CO}_{2}$ ice from the observed spectra of ice mantles, and what is the expected appearance of $\mathrm{LO}$ modes in the spectra of amorphous $\mathrm{CO}_{2}$ ice?

\section{Results and Discussion}

We first present the results obtained with the new Interstellar Astrochemistry Chamber (ISAC) at the Center of Astrobiology (CAB) (17), as described in Materials and Methods. Fig. 1 displays transmission IR spectra of two sets of samples focused on the spectral windows of the antisymmetrical stretch $\left(\nu_{3}\right.$, Left) and bending $\left(\nu_{2}, R i g h t\right)$ motions of $\mathrm{CO}_{2}$. This figure also shows how the profiles of the corresponding IR bands change with the increasing thickness of the sample, from the thinnest one [ 2 monolayers (ML), Fig. 1 (Lower)] to the thickest one [360 ML, Fig. 1 (Upper)]. Whereas for the 2-ML ice, the band frequencies may be affected by interaction with the potassium bromide substrate, for a 13-ML sample, the bands agree with the wavenumber positions commonly reported in the literature for thick ices.

The integrated absorptions of these bands allow us to estimate the column density of the deposits, and hence their thickness (18, 19). The strongest feature, at $2,343 \mathrm{~cm}^{-1}(4.27 \mu \mathrm{m})$, is assigned to $\nu_{3}$ of crystalline $\mathrm{CO}_{2}$, but the origin of the satellite band at 2,328 $\mathrm{cm}^{-1}(4.30 \mu \mathrm{m})$, sometimes referred to as "a low-frequency shoulder" (11) (henceforth, X mode), is still unclear. In previous investigations, it was assigned to perturbed $\mathrm{CO}_{2}$ structures formed when mixed with other components in the ice, such as water $\left(\mathrm{H}_{2} \mathrm{O}\right)(11,20)$, which is usually present in astrophysical dust particles but is seen here in pure $\mathrm{CO}_{2}$ ice. The intensity ratio between the two peaks is clearly dependent on the thickness of the solid, but with the $\mathrm{X}$ mode more prominent at low thicknesses. In Fig. 1 (Right), spectral alterations with sample growth consist of an increase in the high-frequency component of the $\nu_{2}$

Author contributions: R.M.E. and G.M.M.C. designed research; R.M.E., G.M.M.C., G.A.C.-D. Y.R.-L., and B.M. performed research; B.M. analyzed data; and R.M.E. and G.M.M.C. wrote the paper.

The authors declare no conflict of interest.

This article is a PNAS Direct Submission.

${ }^{1}$ To whom correspondence may be addressed. E-mail: rafael.escribano@csic.es or munozcg@ cab.inta-csic.es. 

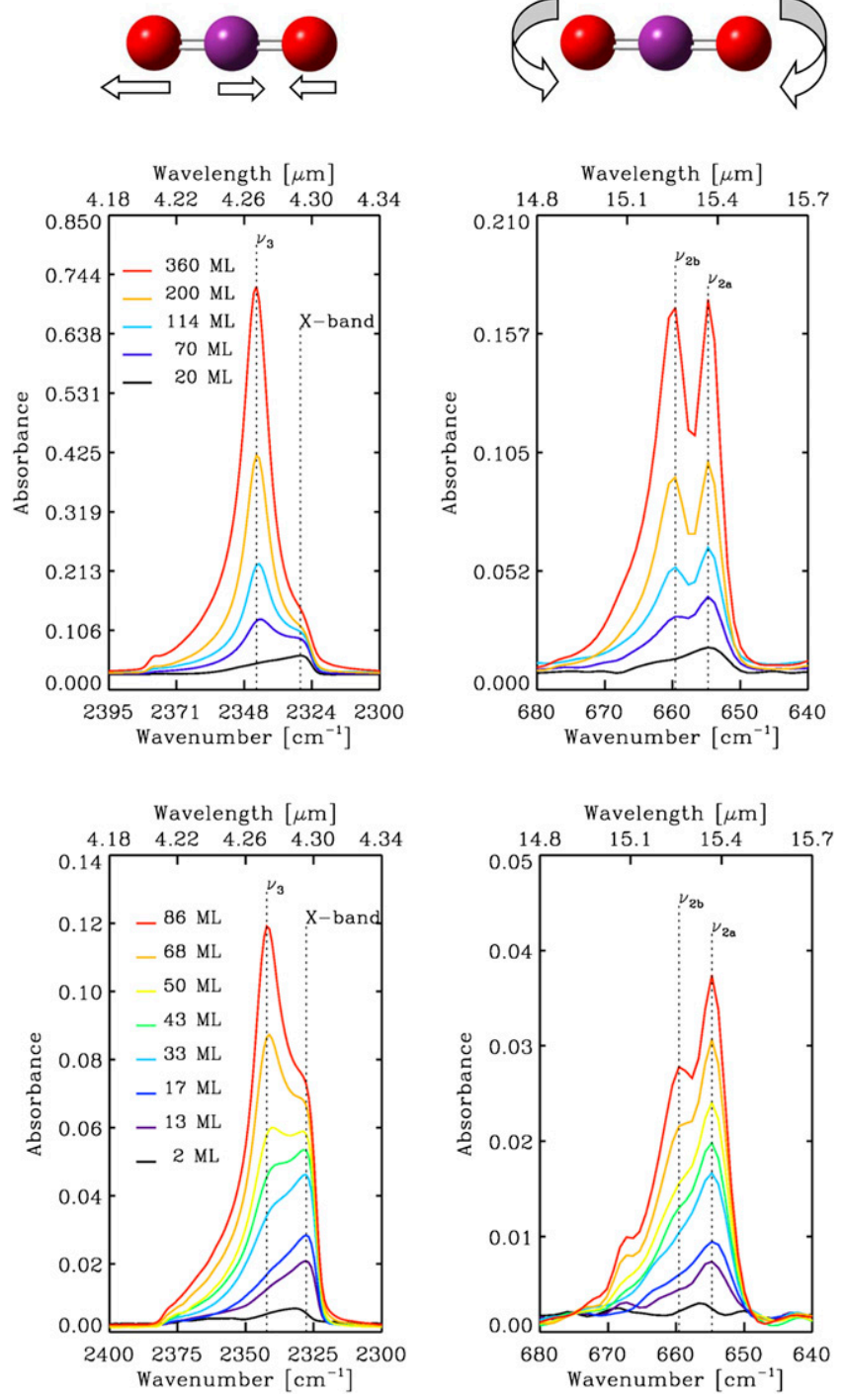

Fig. 1. Transmission spectra of $\mathrm{CO}_{2}$ ice samples deposited at $8 \mathrm{~K}$ for increasing thickness, expressed as monolayer coverage. (Upper) Thicker samples are shown. Spectral regions of $\nu_{3}$ (Left; stretching mode) and $\nu_{2}$ (Right; bending mode) are also shown. Dashed lines indicate the position of the main vibrational modes: the fundamental $\left(\nu_{3}\right)$ and $X$ modes for the stretching region and the so-called "in-plane" and "out-of-plane" modes for the bending region, which are more precisely characterized in this work. A $\mathrm{CO}_{2}$ molecule (red balls, $\mathrm{O}$ atoms; purple ball, $\mathrm{C}$ atom) is schematically represented at $T o p$, where the arrows indicate an asymmetric stretching motion (Left) and a bending motion (Right).

doublet, $\nu_{2 \mathrm{~b}}$, at $660 \mathrm{~cm}^{-1}(15.15 \mu \mathrm{m})$, with respect to the lowfrequency part, $\nu_{2 \mathrm{a}}$, at $655 \mathrm{~cm}^{-1}(15.3 \mu \mathrm{m})$. For the thickest ices, the spectrum resembles the well-known double-peaked profile that is characteristic of pure and crystalline $\mathrm{CO}_{2}$ ice. In our experiments, where $\mathrm{CO}_{2}$ is the only species in the ice, these observations can be rationalized in terms of a crystallization process without requiring the presence of water or other molecular components that lead to a single-band profile near 655 $\mathrm{cm}^{-1}$. Indeed, Fig. 1 shows that the depositions of a few ice monolayers of pure $\mathrm{CO}_{2}$ also display a single band at that position. Additional experiments and theoretical models described below support this assumption.

The substrate where the ices are deposited can be warmed up until complete sublimation of the sample occurs at $\sim 85$ K. Fig. 2 shows spectral variations taking place on a $10-\mathrm{K}$ deposit during warm-up at a rate of $1 \mathrm{~K} \cdot \mathrm{min}^{-1}$. This slow warming induces crystallization of amorphous structures, which is practically attained for $\mathrm{CO}_{2}$ at $\sim 30 \mathrm{~K}$ because there are no significant spectral variations above that temperature. The spectra show that the changes undergone in the lapse of temperature between $10 \mathrm{~K}$ and $30 \mathrm{~K}$ resemble those observed along the build-up of the samples described above. Thus, the warm-up of the sample to $\sim 30 \mathrm{~K}$ by heating the substrate stimulates an effect similar to the accumulation of successive layers at a lower temperature. This process was studied in great detail by Schulze and Abe (21). Complete crystallization, however, is only achieved by thermal annealing. Without warm-up, even the thickest ice sample deposited, 360 ML, still displays a shoulder at $2,328 \mathrm{~cm}^{-1}$ and an intensity ratio of the bending modes that is indicative of not fully crystalline ice.

Transmission spectroscopy of thin films with a normal incidence to the substrate, as performed in the experiments described above, is sensitive to vibrations taking place in the plane of the substrate or with vector components in that plane. Using the experimental set-up available at the Instituto de Estructura de la Materia (IEM), we have recorded reflection-absorption IR (RAIR) spectra with a $75^{\circ}$ incidence angle and radiation polarized in the plane of propagation of the incident radiation (P-polarized RAIR spectra). This technique complements transmission spectroscopy in that it allows observation of vibrations in a normal direction to the substrate or with vector components along that direction (22). Fig. 3 presents P-polarized RAIR spectra of a sample deposited at $14 \mathrm{~K}$ with thickness growing between $6 \mathrm{ML}$ and $36 \mathrm{ML}$. Black dashed lines indicate the position of the $\nu_{3}$ and $\mathrm{X}$ bands in Fig. 3 (Left) and of the $\nu_{2 \mathrm{~b}}, \nu_{2 \mathrm{a}}$ pair in Fig. 3 (Right), as in Figs. 1 and 2. A red dashed line in Fig. 3 shows the position of the LO modes associated with $\nu_{3}$ and $\nu_{2}$, as measured in a transmission experiment with a $30^{\circ}$ incidence angle of a crystalline $\mathrm{CO}_{2}$ thin sample (additional details are provided in Materials and Methods). Thus, the trend of the spectra in Fig. $3 A$ and $B$, where the longitudinal modes sharpen and approach the LO maximum, indicates again that the crystallization process is taking place in the sample during its growth. In addition, it can be seen that whereas $\nu_{3}$ and $\nu_{2 \mathrm{a}}$ become more distinguishable along the series, the $\mathrm{X}$ band is completely missing on these spectra, as is the $\nu_{2 b}$ component. In consequence, we can conclude that the corresponding modes, $\mathrm{X}$ and $\boldsymbol{\nu}_{2 \mathrm{~b}}$, oscillate essentially in a plane parallel to the substrate.

To complement these experiments, we have made computer models of amorphous and crystalline $\mathrm{CO}_{2}$. Pure crystals are straightforward to simulate using the available geometry from X-ray diffraction (23) measurements. On the contrary, the main difficulty in modeling amorphous $\mathrm{CO}_{2}$ is to devise a sufficiently amorphous initial structure. Low-temperature crystalline $\mathrm{CO}_{2}$ has a density of $1.78 \mathrm{~g} / \mathrm{cm}^{3}$, but the amorphous ice is more porous, with densities that can change within a range of values (21). Thus, to realize a low-density amorphous model, we took an ensemble of $32 \mathrm{CO}_{2}$ molecules initially arranged as a set of eight unit cells of the crystal, enlarged the volume of the cell to a selected size to reduce the density, and subjected the sample to increasing temperature to jolt the molecules away from their crystalline equilibrium structure by means of molecular dynamics (MD) simulations. In this way, we prepared models with densities of $1.0 \mathrm{~g} / \mathrm{cm}^{3}$ and $1.3 \mathrm{~g} / \mathrm{cm}^{3}$. The strength of the intermolecular forces in the crystal for $\mathrm{CO}_{2}$ is such that even for these low densities, we had to increase the temperature of the MD reactor to unrealistic values $(500 \mathrm{~K})$ before an amorphous, or fully disordered, structure could be reached.

The solids thus designed were treated with the SIESTA method (24-26), a well-known and widely used computational tool specifically designed for solids and repetitive units. The initial structures can be relaxed until a well-defined minimum is found on the potential energy surface, for which the vibrational spectrum can be predicted. The unit cell of $\mathrm{CO}_{2}$ crystals belongs 

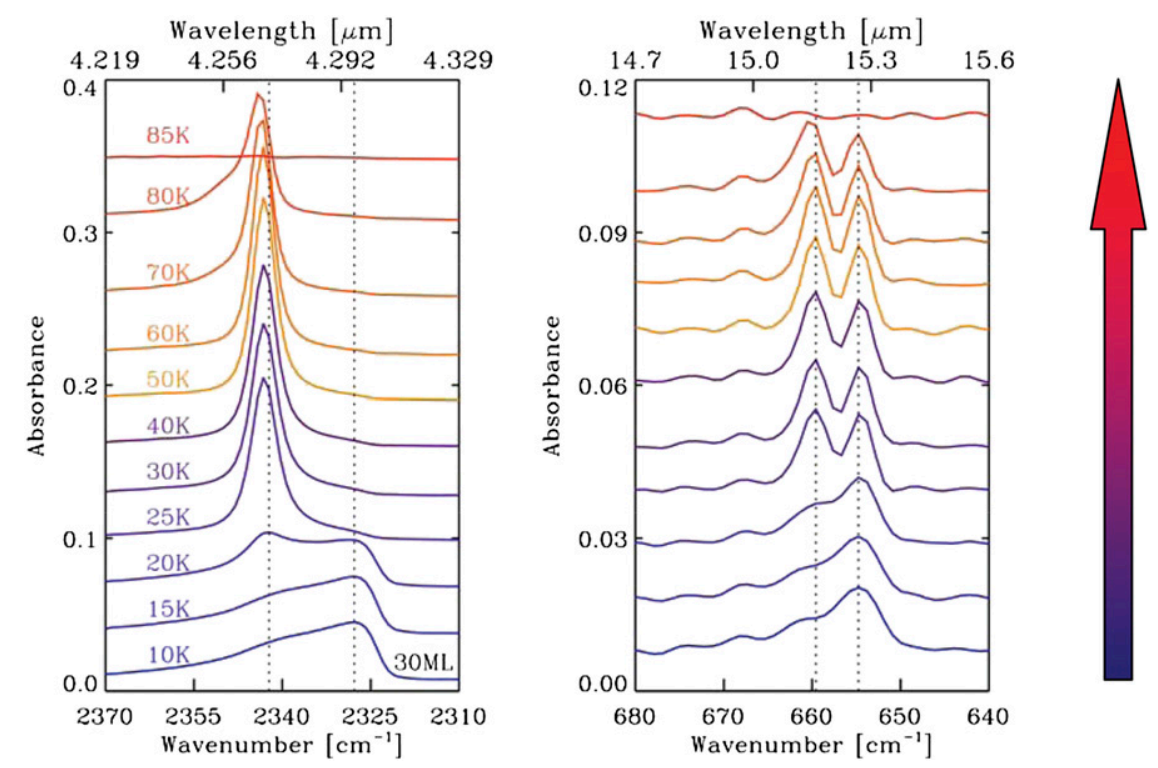

Fig. 2. Thermal variation of a $30-\mathrm{ML}$ sample from the deposition temperature at $10 \mathrm{~K}$ to complete sublimation at $85 \mathrm{~K}$. Spectra are offset for clarity. The arrow on the right indicates increasing temperature along the experiment.

to the Pa3 space group and contains four molecules arranged at alternate corners of a cubic structure. From the IR-active vibrational modes of the four molecules, crystal modes are generated with different IR activity (27): The doubly degenerate $\nu_{2}$ vibrations split into three independent modes, two of $F_{u}$ symmetry and one of $E_{u}$ symmetry, and $\nu_{3}$ yields one $F_{u}$ mode and one $A_{u}$ mode. Of these, only the $F_{u}$ vibrations are IR-active in the crystal, and they are usually labeled $\nu_{2 \mathrm{a}}, \nu_{2 \mathrm{~b}}$, and $\nu_{3}$, respectively. All these vibrations are predicted in our models. Fig. 4 presents a composite of schematic representations of crystalline and amorphous structures with the corresponding predicted spectra, which appear with a red shift from the experimental measurements of $\sim 50 \mathrm{~cm}^{-1}$ and $43 \mathrm{~cm}^{-1}$ for the $\nu_{3}$ and $\nu_{2}$ regions, respectively, that can be attributed to imperfections in the model.

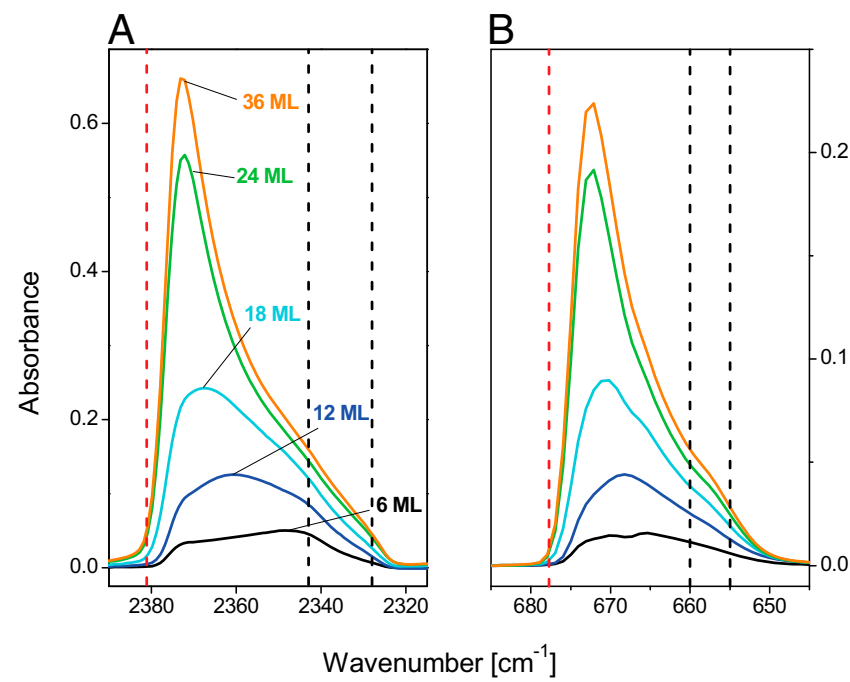

Fig. 3. RAIR spectra of $\mathrm{CO}_{2}$ samples deposited at $14 \mathrm{~K}$ with a growing thickness between 6 and $36 \mathrm{ML}$. Black dashed lines mark the wavenumber position, in decreasing frequency, of the $\nu_{3}$ and $X$ modes $(A)$ and of the $\nu_{2} \mathrm{~b}$ and $\nu_{2 a}$ components $(B)$. Red dashed lines indicate the observed wavenumber for the LO modes in transmission spectra of pure crystals at a $30^{\circ}$ incidence (19).
The aim of these theoretical calculations was not to achieve a perfect match in frequency with the experiment but to develop a joint model for crystalline and amorphous $\mathrm{CO}_{2}$ that provides explanations for the special characteristics of their IR spectra.

Several aspects of the calculations deserve special mention. First, the IR-inactive $\mathrm{A}_{\mathrm{u}}$ component of $\nu_{3}$ is calculated with a red shift of $14 \mathrm{~cm}^{-1}$ from $\nu_{3}$, that is, coincident with the red shift of the $\mathrm{X}$ band from the observed $\nu_{3}$. The symmetry restrictions that force the $\mathrm{A}_{\mathrm{u}}$ mode to cancel along the crystal do not apply to the amorphous structures, where this band is consequently allowed. Thus, the unexplained origin of the $\mathrm{X}$ band seems clear: It is a pure $\mathrm{CO}_{2}$ mode that can only be seen in IR spectra when amorphous or disordered structures are present. Furthermore, the fact that the $\mathrm{X}$ band near $2,328 \mathrm{~cm}^{-1}$ was not detected in IR spectra of dense clouds and circumstellar regions, where the other $\mathrm{CO}_{2}$ ice bands were observed, indicates that, so far, there is no evidence for the presence of pure and amorphous $\mathrm{CO}_{2}$ ice in these environments.

Second, when the Cartesian components of the $\nu_{2 \mathrm{a}}$ and $\nu_{2 \mathrm{~b}}$ modes are analyzed, their predicted orientation sets $\nu_{2 \mathrm{a}}$ in a tilted direction and sets $\nu_{2 \mathrm{~b}}$ within the $x-y$ plane. If we assume that the substrate defines the $x-y$ plane, $\nu_{2 \mathrm{a}}$ would be oblique to the substrate and $\nu_{2 \mathrm{~b}}$ would be in-plane, in agreement with the observed activity of these modes in normal incidence transmission and in P-polarized RAIR. Vectors depicting these modes are represented in Fig. 4 (Top).

Fig. 4 also shows how features in the spectra of the amorphous models evolve toward those of the crystal as the density is increased: The $\mathrm{X}$ band, at a frequency $c a .14 \mathrm{~cm}^{-1}$ lower than that of $\nu_{3}$, becomes weaker and $\nu_{3}$ becomes stronger in this sequence, and in the $\nu_{2}$ region, the $\nu_{2 \mathrm{a}}$ component gains intensity and $\nu_{2 \mathrm{~b}}$ starts to form.

This work has implications for the interpretation of ice mantle observations in the mid-IR spectral range. The fact that no band around $4.30 \mu \mathrm{m}\left(2,328 \mathrm{~cm}^{-1}\right)$ was clearly detected in the ice spectra toward dense clouds and protostars confirms that pure $\mathrm{CO}_{2}$ ice is formed at temperatures above $25 \mathrm{~K}$. This agrees with recent models of ice mantle evolution in protostellar envelopes, where pure and crystalline $\mathrm{CO}_{2}$ ice is formed after distillation of $\mathrm{CO}$ at $\sim 20-30 \mathrm{~K}$, and perhaps also at higher temperatures, leading to $\mathrm{CO}_{2}$ inclusions in the water and ice matrix $(9,10)$. The $\mathrm{CO}_{2} /$ $\mathrm{H}_{2} \mathrm{O}$ ice mixture displays a single band around $15.3 \mu \mathrm{m}\left(655 \mathrm{~cm}^{-1}\right)$ that is extensively used as one of the components to fit the bending modes of $\mathrm{CO}_{2}$ ice observed $(9,10,20)$. Fig. 1 shows 

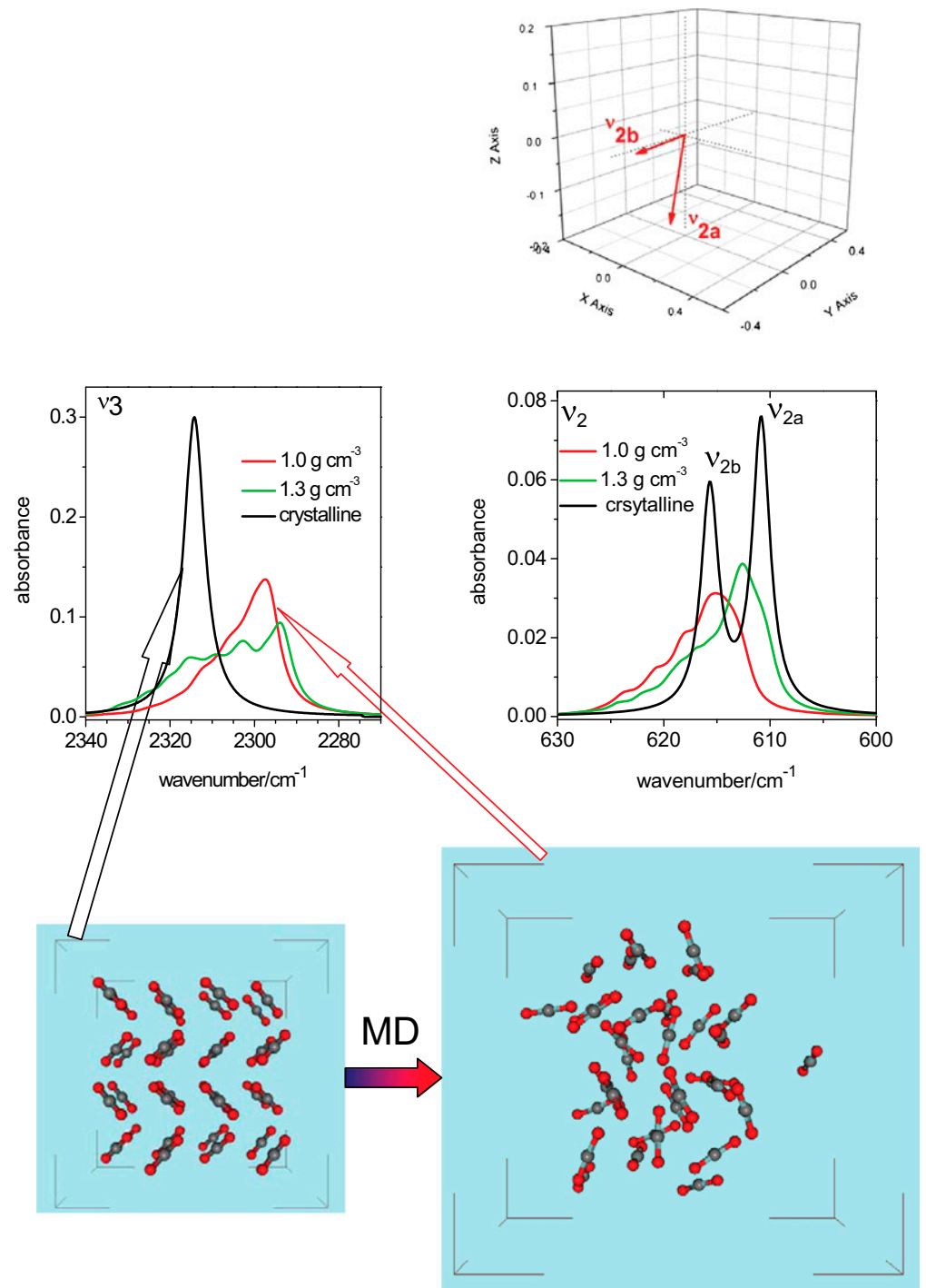

Fig. 4. (Bottom) Theoretical models for crystalline (Left) and amorphous (Right) $\mathrm{CO}_{2}$ solids; the amorphous solid is obtained from the crystalline solid by $\mathrm{MD}$ annealing. (Middle) Calculated spectra of crystalline (black) and amorphous $\mathrm{CO}_{2}$ with densities of $1.0 \mathrm{~g} \cdot \mathrm{cm}^{-3}$ and $1.3 \mathrm{~g} \cdot \mathrm{cm}^{-3}$ (in red and green, respectively). (Top) Predicted orientation of the $\nu_{2 \mathrm{a}}$ and $\nu_{2 \mathrm{~b}}$ components of the bending. The $\nu_{2 \mathrm{~b}}$ component is practically contained in the $x-y$ plane.

that a single band at a similar position is also obtained by depositing a few ice monolayers (less than $\sim 50 \mathrm{ML}$ ) of pure $\mathrm{CO}_{2}$ if the ice temperature does not exceed $\sim 25 \mathrm{~K}$. However, if we accept that the $\mathrm{CO}_{2}$ observed in the ice mantles has experienced temperatures above $25 \mathrm{~K}$, as the absence of a $4.30-\mu \mathrm{m}\left(2,328-\mathrm{cm}^{-1}\right)$ band indicates and current astrophysical models suggest $(9,10)$, the detection of the $15.3-\mu \mathrm{m}\left(655-\mathrm{cm}^{-1}\right)$ component must require mixing of $\mathrm{CO}_{2}$ with species like $\mathrm{H}_{2} \mathrm{O}$ in the polar ice phase. On the other hand, the detection of the two bending modes at $15.15 \mu \mathrm{m}$ and $15.3 \mu \mathrm{m}$ in other lines of sight, for ice mantles in which $\mathrm{CO}_{2}$ is mixed with $\mathrm{H}_{2} \mathrm{O}$ and other species, implies that segregation temperatures above $25 \mathrm{~K}$ were reached, as proposed by Gerakines et al. (20).

In summary, our detailed study of pure and solid $\mathrm{CO}_{2}$ supports the current scenario for the formation of pure $\mathrm{CO}_{2}$ ice by two processes. These processes consist of $\mathrm{CO}_{2}$ segregation out of a $\mathrm{CO}_{2}-\mathrm{H}_{2} \mathrm{O}$ mixture at high temperature $(50-80 \mathrm{~K})$, on the one hand, and $\mathrm{CO}$ evaporation from a $\mathrm{CO}_{2}-\mathrm{CO}$ mixture, leaving pure $\mathrm{CO}_{2}$ behind, at a lower temperature $(20-30 \mathrm{~K})$, on the other hand $(9,10,20,28)$. Accordingly, pure $\mathrm{CO}_{2}$ ice present in ice mantles has experienced a temperature of at least $20-30 \mathrm{~K}$. We show that at around $25 \mathrm{~K}$, the band near $4.30 \mu \mathrm{m}\left(2,328 \mathrm{~cm}^{-1}\right)$ tracing pure and highly amorphous $\mathrm{CO}_{2}$ ice disappears. As a consequence, pure $\mathrm{CO}_{2}$ ice in space cannot be completely amorphous. Further evidence is provided by the absence of this band in the published spectra of $\mathrm{CO}_{2}$ ice observed toward dense clouds and protostars. An example is shown in Fig. 5, where our laboratory spectra are compared with those of Elias 16 (29), a field star probing the ice in a dark cloud.

\section{Materials and Methods}

ISAC at the CAB. A set of experiments was performed using the ISAC of the $C A B$, as described in detail by Muñoz Caro et al. (17). The optimum vacuum conditions, base pressure in the range of $10^{-11} \mathrm{mbar}$, and use of a needle valve allowed the deposition of $\mathrm{CO}_{2}$ ice at a very low rate. The column density of the deposited ice was calculated according to the formula

$$
N=\frac{\int_{\text {band }} \tau_{\nu} d \nu}{A},
$$

where $N$ is the column density (in square centimeters), $\tau_{\nu}$ is the optical depth of the band, $d_{\nu}$ is the wavenumber differential (in $\mathrm{cm}^{-1}$ ), and $A$ is the band strength (in $\mathrm{cm} \cdot$ molecule $^{-1}$ ). The integrated absorbance is equal to $0.43 \times \tau$, 


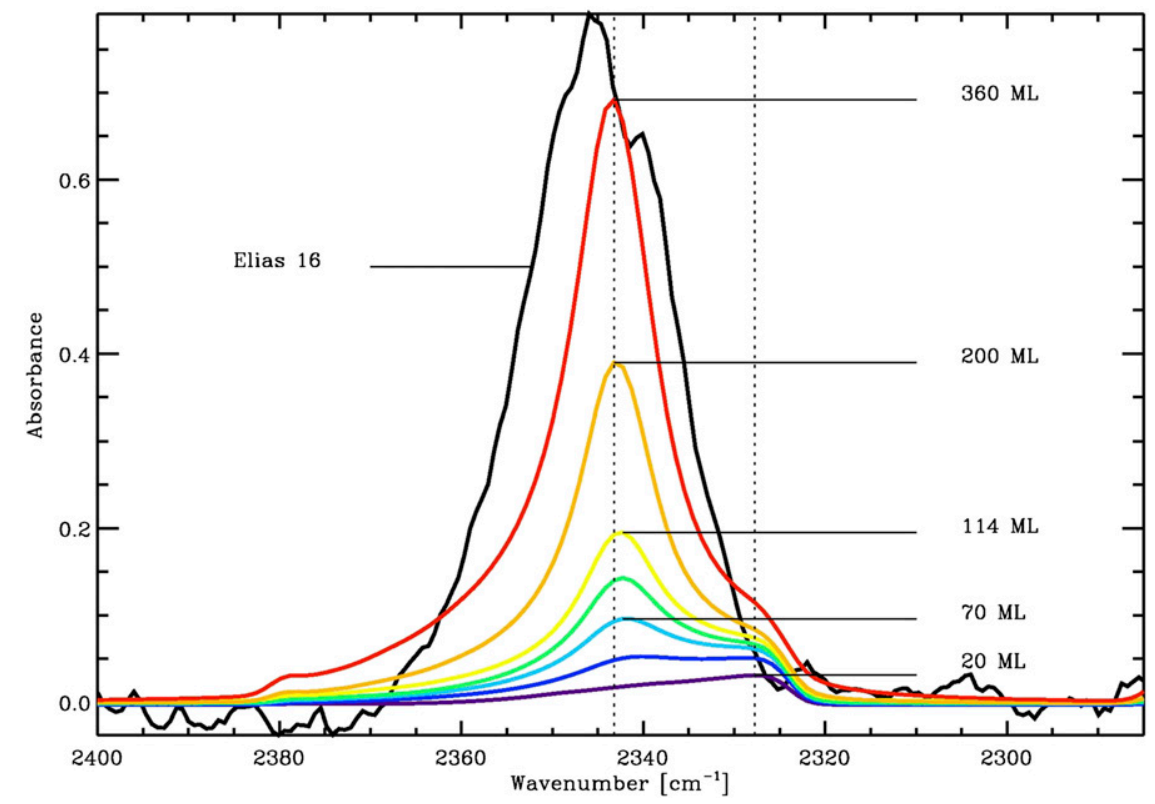

Fig. 5. Comparison of the $2,343-\mathrm{cm}^{-1}(4.27-\mu \mathrm{m})$ band toward Elias 16, probing a dark cloud environment, with the laboratory measurements shown in Fig. 1 . The optical depth of Elias 16 is represented in the absorbance scale after dividing by $\log (10)$. The $X$ band at $2,328 \mathrm{~cm}^{-1}(4.3 \mu \mathrm{m})$, which is seen as a shoulder in the 360-ML ice sample, is not present in the astrophysical observation. [Elias 16 spectrum data from ref. 29.]

where $\tau$ is the integrated optical depth of the band. The adopted band strengths for the $\nu_{3}$ stretching $\left(\right.$ at $2,343 \mathrm{~cm}^{-1}$ ) and $\nu_{2}$ degenerate bending (at $660 \mathrm{~cm}^{-1}$ and $\left.665 \mathrm{~cm}^{-1}\right)$ modes of $\mathrm{CO}_{2}$ were $\mathrm{A}\left(\mathrm{CO}_{2}, \nu_{3}\right)=7.6 \times 10^{-17}$ $\mathrm{cm} \cdot$ molecule $^{-1}(18)$ and $\mathrm{A}\left(\mathrm{CO}_{2}, \nu_{2}\right)=1.1 \times 10^{-17} \mathrm{~cm} \cdot$ molecule $^{-1}(19)$, respectively. The ice was warmed at a heating rate of $1 \mathrm{~K} \cdot \mathrm{min}^{-1}$. Quadrupole MS was used to detect the desorbing $\mathrm{CO}_{2}$ in the ISAC chamber during ice warm-up (i.e., the partial pressure increases for $m / z=44$, corresponding to the ion molecule of $\mathrm{CO}_{2}$ ).

Assuming that $1 \times 10^{15}$ molecules $\mathrm{cm}^{-2}$ is about $1 \mathrm{ML}$ of coverage, the deposition flow is estimated as $0.014 \mathrm{ML} / \mathrm{s}$. After deposition of the ice, the cryostat was rotated $90^{\circ}$ to face the IR radiation at normal incidence. FTIR spectra were recorded with a spectral resolution of $2 \mathrm{~cm}^{-1}$.

RAIR at the IEM. Grazing angle RAIR spectra were recorded at the IEM with an experimental set-up described in more detail elsewhere $(30,31)$. Briefly, it consists of a high-vacuum chamber, with a background pressure in the range of $10^{-8}$ mbar, provided with a closed cycle helium cryostat with its cold finger in close contact with a gold plate onto which the gases are condensed. The vacuum chamber is coupled through a purged pathway to a Vertex70 Bruker FTIR spectrometer in a $75^{\circ}$ grazing angle configuration. The RAIR spectra were recorded at a resolution of $2 \mathrm{~cm}^{-1}$ using a liquid nitrogen-cooled mercurium cadmium telluride (MCT) detector and accumulating 500 scans. Ice layers of $\mathrm{CO}_{2}$ were generated by introducing a controlled flow of pure $\mathrm{CO}_{2}$ to backfill the chamber to a pressure of about $1 \times 10^{-7} \mathrm{mbar}$, which condenses on the cold substrate at $14 \mathrm{~K}$. The substrate can be warmed up to anneal the samples.

As mentioned above, transmission spectroscopy of thin films with a normal incidence to the substrate responds to vibrations taking place in the plane of the substrate, corresponding to the TO branch of solids. When the incident radiation is tilted, spectral features are expected to accompany the TO modes, corresponding to long-range dipole-dipole interactions through the sample, which become optically active by coupling with the nonnormal components of the radiation. They are called LO modes, and they always appear at higher frequencies than the TO modes. The theory describing the properties of these surface or longitudinal modes, and the spectral features that can appear between the LO and TO frequencies, have been extensively described in the literature $(27,32-36)$. The magnitude of the LO-TO separation is given by

$$
\omega_{n}^{2}=\omega_{0}^{2}+\frac{4 \pi}{\varepsilon V}\left(\frac{\partial \mu}{\partial Q}\right)^{2} \Omega_{\alpha}
$$

where $\omega_{n}$ indicates the frequency of the longitudinal modes and $\omega_{0}=\omega_{\text {TO }}$ Here, $V$ is the volume of the sample, the parameter $\Omega_{\alpha}$ can vary between 0 and 1 , the dielectric constant of the crystal is $\varepsilon$, and $\partial \mu / \partial Q$ represents the derivative of the transition dipole moment with respect to the $Q$ normal mode. For single crystals, $V$ is the unit cell volume, and this expression gives the maximum value of $\omega_{n}=\omega_{\mathrm{LO}}$. For pure crystals shaped as thin slabs and with a tilted incidence angle, the theory predicts only two peaks at exactly the LO and TO frequencies; broad absorptions appearing between them are ascribed to randomly oriented polycrystallites or to the amorphous structure of the sample $(27,32,33)$. For irregular crystals or amorphous solids, $\omega_{n}$ can take values between $\omega_{\mathrm{LO}}$ and $\omega_{\mathrm{TO}}$, as seen on the RAIR spectra recorded at the IEM (Fig. 3).

The characteristics of longitudinal modes in amorphous crystals become evident in Fig. 6 . Fig. 6 (Upper) shows transmission spectra of a sample
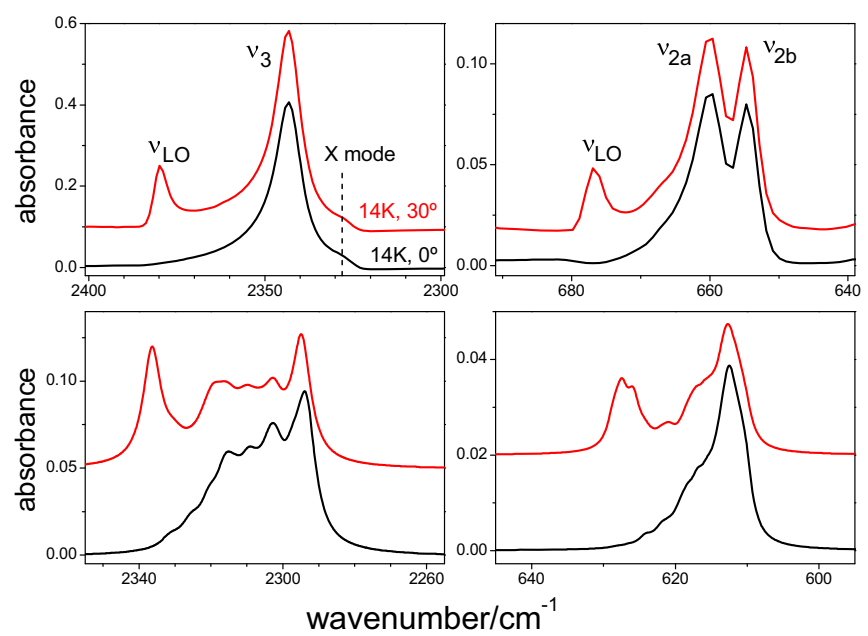

Fig. 6. (Upper) IR transmission spectra of $\mathrm{CO}_{2}$ deposited at $14 \mathrm{~K}$ at a normal incidence (black) and at a $30^{\circ}$ incidence (red). Due to the large thickness of the sample (around $200 \mathrm{ML}$ ), these spectra display features characteristic of partially crystalline ice. Tilted incidence spectra are offset on the vertical axis for clarity. (Lower) Calculated $\mathrm{CO}_{2}$ spectra: amorphous without (black) and with (red) LO/TO. A direct frequency comparison with the experimental spectra should not be attempted due to imperfections in the theoretical model (main text), which produce red shifts of $\sim 50 \mathrm{~cm}^{-1}$ and $43 \mathrm{~cm}^{-1}$ for the $\nu_{3}$ and $\nu_{2}$ regions, respectively. 
deposited at $14 \mathrm{~K}$ recorded with normal (black trace) and oblique (red) incidence light. The sample displays some crystalline character due to the layer accumulation even at this low temperature, as evidenced by the splitting of the $\nu_{2}$ modes. The LO modes appear blue-shifted with respect to the transverse modes. Fig. 6 (Lower) presents calculated spectra of a $1.3-\mathrm{g} \cdot \mathrm{cm}^{-3}$ amorphous model, showing the important intensity transfer that takes place when the appropriate electrostatic dipole-dipole interactions are taken into account (28). It can be seen that our calculations predict a LO-TO displacement in good agreement with the observed values for amorphous $\mathrm{CO}_{2}$.

Theoretical Method. The specific method and parameters used for our SIESTA calculations follow those used in a previous study by our group (22). Specifically, we have performed density functional theory calculations using PerdewBurke-Ernzerhof exchange-correlation functionals (37) of the generalized gradient approximation. The basis set for the valence electrons was double-Z polarized (38). The force tolerance parameter was set to $0.5 \mathrm{meV} \cdot \AA^{-1}$ for the amorphous systems and to $0.05 \mathrm{meV} \cdot \AA^{-1}$ for the crystalline species. The stress tolerance was set $0.1 \mathrm{GPa}$, a fairly large value but reasonable for our fixed-cell calculations. Born effective charges for the prediction of absorption intensities have been evaluated by numerical derivation of the macroscopic polarization, with a displacement step of 0.02 Bohr, the same value as in

1. Sloan ED (2003) Fundamental principles and applications of natural gas hydrates. Nature 426(6964):353-359.

2. Park $Y$, et al. (2006) Sequestering carbon dioxide into complex structures of naturally occurring gas hydrates. Proc Natl Acad Sci USA 103(34):12690-12694.

3. Cruikshank DP, et al. (2010) Carbon dioxide on the satellites of Saturn: Results from the Cassini VIMS investigation and revisions to the VIMS wavelength scale. Icarus 206(2):561-572.

4. Zasowski G, et al. (2010) Spitzer infrared spectrograph observations of class I/II objects in Taurus: Composition and thermal history of the circumstellar ices. Astrophys 694(1):459-478.

5. Gibb EL, Whittet DCB, Boogert ACA, Tielens AGGM (2004) Interstellar ice: The infrared space observatory legacy. Astrophys J Supp/ Ser 151(1):35-73.

6. Aikawa Y, et al. (2012) AKARI observations of ice absorption bands towards edge-on young stellar objects. Astron Astrophys 538:A57-A69.

7. Hodyss R, Johnson PV, Orzechowska GE, Goguen JD, Kanik I (2008) Carbon dioxide segregation in mixed $\mathrm{CO}_{2}: \mathrm{H}_{2} \mathrm{O}$ ices. Icarus 194(2):830-836.

8. Bartels-Rausch T, et al. (2012) Ice structures, patterns, and processes: A view across the icefields. Rev Mod Phys 84(2):885-944.

9. Pontoppidan KM, et al. (2008) The c2d Spitzer spectroscopic survey of ices around low-mass young stellar objects. II. $\mathrm{CO}_{2}$. Astrophys J 678(2):1005-1031.

10. Kim HJ, Evans NJ II, Dunham MM, Lee J-E, Pontoppidan KM (2012) $\mathrm{CO}_{2}$ ice toward low-luminosity embedded protostars: Evidence for episodic mass accretion via chemical history. Astrophys J 758(1):38-52.

11. Sandford SA, Allamandola LJ (1990) The physical and infrared spectral properties of $\mathrm{CO}_{2}$ in astrophysical ice analogs. Astrophys $J$ 355(1):357-372.

12. Dartois E, Demyk K, d'Hendecourt L, Ehrenfreund P (1999) Carbon dioxide-methanol intermolecular complexes in interstellar grain mantles. Astron Astrophys 351(3): 1066-1074.

13. Bernstein MP, Cruikshank DP, Sandford SA (2005) Near-infrared laboratory spectra of solid $\mathrm{H}_{2} \mathrm{O} / \mathrm{CO}_{2}$ and $\mathrm{CH}_{3} \mathrm{OH} / \mathrm{CO}_{2}$ ice mixtures. Icarus 179(2):527-534.

14. van Broekhuizen FA, Groot IMN, Fraser HJ, van Dishoeck EF, Schlemmer S (2006) Infrared spectroscopy of solid $\mathrm{CO}-\mathrm{CO}_{2}$ mixtures and layers. Astron Astrophys 451(2): 723-731.

15. Maté B, Gálvez O, Herrero VJ, Escribano R (2009) Infrared spectra and thermodynamic properties of $\mathrm{CO}_{2} /$ methanol ices. Astrophys J 690(1):486-495.

16. Whalley E, Klug DD (1979) Comment on TO-LO splitting in disordered phases of ice. $J$ Chem Phys 71(3):1513.

17. Muñoz Caro GM, et al. (2010) New results on thermal and photodesorption of CO ice using the novel InterStellar Astrochemistry Chamber (ISAC). Astron Astrophys 522:A108-A121.

18. Yamada $\mathrm{H}$, Person WB (1964) Absolute infrared intensities of the fundamental absorption bands in solid $\mathrm{CO}_{2}$ and $\mathrm{N}_{2} \mathrm{O}$. J Chem Phys 41(8):2478-2587.

19. Gerakines PA, Schutte WA, Greenberg JM, van Dishoeck E (1995) The infrared band strengths of $\mathrm{H}_{2} \mathrm{O}, \mathrm{CO}$ and $\mathrm{CO}_{2}$ in laboratory simulations of astrophysical ice mixtures. Astron Astrophys 296(3):810-818. the force constants calculation. The electronic contribution to the polarization was calculated using the Berry phase formalism (39), by discrete integration over the points of a grid with dimensions of $5 \times 2 \times 2$.

Initial structures for the amorphous systems were obtained by MD annealing simulations of a 32-molecule ensemble, with a target temperature of $500 \mathrm{~K}$, at 1 -fs steps, and with the same parameters for the SIESTA method as described above, where appropriate. At the end of the run, the geometrical configuration of the system was taken as the starting point for relaxation and prediction of the spectra. A graphical representation of the crystal and of the $1.0-\mathrm{g} \cdot \mathrm{cm}^{-3}$ amorphous structure is shown in Fig. 4, together with the IR predicted spectra. Densities of $1.0 \mathrm{~g} \cdot \mathrm{cm}^{-3}$ and $1.3 \mathrm{~g} \cdot \mathrm{cm}^{-3}$ of the amorphous systems correspond to original cubic unit cells with side lengths of $6.630 \AA$ and $6.079 \AA$, respectively (5.624 $\AA$ for the crystal).

ACKNOWLEDGMENTS. We thank E. Dartois, Y. Aikawa, and D. C. B. Whittet for their kind support in the astrophysical aspects of this work; P. Gutiérrez for valuable suggestions; and M. A. Moreno for technical support. Most of the calculations were carried out at West-Grid, Compute/Calcul Canada. This work was supported by Projects FIS2010-16455, PR2010-0012, AYA2011 29375, and CSD2009-00038 of the Spanish Ministry of Education, and JAEDoc Consejo Superior de Investigaciones Científicas Programme (Y.R.-L.).

20. Gerakines PA, et al. (1999) Observations of solid carbon dioxide in molecular clouds with the infrared space observatory. Astrophys J 522(1):357-377.

21. Schulze W, Abe $\mathrm{H}(1980)$ Density, refractive index and sorption capacity of solid $\mathrm{CO}_{2}$ layers. Chem Phys 52(3):381-388

22. Maté $\mathrm{B}$, et al. (2008) Ices of $\mathrm{CO}_{2} / \mathrm{H}_{2} \mathrm{O}$ mixtures. Reflection-absorption IR spectroscopy and theoretical calculations. J Phys Chem A 112(3):457-465

23. Simon A, Peters K (1980) Single-crystal refinement of the structure of carbon dioxide. Acta Crystallogr B 36:2750-2751.

24. Soler JM, et al. (2002) The SIESTA method for ab initio order-N materials simulation. J Phys Condens Matter 14(11):2745-2779.

25. Ordejón $\mathrm{P}$, Artacho $\mathrm{E}$, Soler JM (1996) Self-consistent order- $\mathrm{N}$ density-functional calculations for very large systems. Phys Rev B Condens Matter 53(16):R10441-R10444.

26. Gonze X, Lee C (1997) Dynamical matrices, Born effective charges, dielectric permittivity tensors, and interatomic force constants from density-functional perturbation theory. Phys Rev B Condens Matter 55(16):10355-10368.

27. Decius JC, Hexter RM (1977) Molecular Vibrations in Crystals (McGraw-Hill, New York).

28. Fayolle EC, Öberg KI, Cuppen HM, Visser R, Linnartz $\mathrm{H}$ (2011) Laboratory $\mathrm{H}_{2} \mathrm{O}: \mathrm{CO}_{2}$ ice desorption data: Entrapment dependencies and its parameterization with an ex tended three-phase model. Astron Astrophys 529:A74-A84.

29. Whittet $D C B$, et al. (1998) Detection of abundant $\mathrm{CO}_{2}$ ice in the quiescent dark cloud medium toward Elias 16. Astrophys J 498(2):L159-L163.

30. Maté B, Medialdea A, Moreno MA, Escribano R, Herrero VJ (2003) Experimental studies of amorphous and polycrystalline ice films using FT-RAIRS. J Phys Chem B 107(40):11098-11108.

31. Carrasco E, et al. (2002) A cryostat for low-temperature spectroscopy of condensable species. Rev Sci Instrum 73(10):3469-3473.

32. Fox D, Hexter RM (1964) Crystal shape dependence of exciton states in molecular crystals. J Chem Phys 41(4):1125-1139.

33. Ovchinnikov MA, Wight CA (1993) Inhomogeneous broadening of infrared and Raman spectral bands of amorphous and polycrystalline thin films. J Chem Phys 99(5): 3374-3379.

34. Devlin JP, Yinnon CA, Buch V (2009) Spectroscopic evidence for cyclical aggregation and coalescence of molecular aerosol particles. Phys Chem Chem Phys 11(36): 7819-7825.

35. Signorell $\mathrm{R}$ (2003) Verification of the vibrational exciton approach for $\mathrm{CO}_{2}$ and $\mathrm{N}_{2} \mathrm{O}$ Nanoparticles. J Chem Phys 118(6):2707-2715.

36. Signorell $R$, Jetzki $M$, Kunzmann $M$, Ueberschaer $R$ (2006) Unravelling the origin of band shapes in infrared spectra of $\mathrm{N}_{2} \mathrm{O}_{-}{ }^{12} \mathrm{CO}_{2}$ and ${ }^{12} \mathrm{CO}_{2}{ }^{-13} \mathrm{CO}_{2}$ ice particles. $J$ Phys Chem 110(9):2890-2897.

37. Perdew JP, Burke K, Ernzerhof M (1996) Generalized Gradient Approximation Made Simple. Phys Rev Lett 77(18):3865-3868

38. Fernández-Serra MV, Artacho E (2006) Electrons and hydrogen-bond connectivity in liquid water. Phys Rev Lett 96(1):016404-1-016404-4.

39. King-Smith RD, Vanderbilt D (1993) Theory of polarization of crystalline solids. Phys Rev B Condens Matter 47(3):1651-1654. 